\title{
Influence of Vocal and Verbal Cues on Ratings of Interview Anxiety and Interview Performance
}

\author{
Ryan O. Miller \\ Carleton University \\ Brianne L. Gayfer \\ University of Guelph \\ Deborah M. Powell \\ University of Guelph
}

Follow this and additional works at: https://scholarworks.bgsu.edu/pad

Part of the Human Resources Management Commons, Industrial and Organizational Psychology Commons, and the Other Psychology Commons

How does access to this work benefit you? Let us know!

\section{Recommended Citation}

Miller, Ryan O.; Gayfer, Brianne L.; and Powell, Deborah M. (2018) "Influence of Vocal and Verbal Cues on Ratings of Interview Anxiety and Interview Performance," Personnel Assessment and Decisions: Number 4 : Iss. 2 , Article 3.

DOI: https://doi.org/10.25035/pad.2018.02.003

Available at: https://scholarworks.bgsu.edu/pad/vol4/iss2/3

This Main Article is brought to you for free and open access by the Journals at ScholarWorks@BGSU. It has been accepted for inclusion in Personnel Assessment and Decisions by an authorized editor of ScholarWorks@BGSU. 


\title{
Influence of Vocal and Verbal Cues on RATINGS OF INTERVIEW ANXIETY AND INTERVIEW PeRFoRMANCE
}

\author{
Ryan O. Miller ${ }^{1}$, Brianne L. Gayfer ${ }^{2}$, and Deborah M. Powell ${ }^{2}$ \\ 1. Carleton University \\ 2. University of Guelph
}

ABSTRACT

\begin{abstract}
In two studies, we examined the effect of the presence (versus absence) of vocal cues on judges' ratings of interview anxiety and interview performance. In Study 1, we designed an experiment in which participants rated either a high-anxiety candidate or a low-anxiety candidate and were exposed to either an audio version of the interview or a text-only version. In Study 2, we added a third condition-a text-only version with filler words (um and ah) cleaned out. In two online studies ( $n=72$ and $n=411)$, we found that the highanxiety interviewee was rated higher on observer-rated anxiety and lower on observer ratings of interview performance as compared to the low-anxiety interviewee across both text and audio conditions, which did not support our hypothesis that anxiety would be less detectable when vocal cues were restricted in the text-only condition. Overall, this study provides powerful evidence of the ability of observers to recognize interviewee anxiety and highlights the negative impact of interview anxiety on the perceived interview performance of interviewees by observers.
\end{abstract}

Employment interviews involve meeting strangers, talking about oneself, and being evaluated - all of which can be very anxiety provoking (McCarthy \& Goffin, 2004). Numerous studies have investigated the relationship between interview anxiety and interview performance, and have found a moderate negative correlation (e.g. $r=-.19$ to $r=-.32$; McCarthy \& Goffin, 2004; Feiler \& Powell, 2015). Given that anxiety seems to be related to lower interview performance, it is important to understand how anxiety is manifested in employment interviews.

Research has yet to fully explain the underlying mechanisms responsible for this correlation between anxiety and interview performance. Little is known about why anxious interviewees receive lower interview ratings and what behaviors (or cues) might signal anxiety to interviewers. Several studies have found that interviewers can and do form impressions of interviewees based on their nonverbal (e.g., eye contact), verbal (e.g., filler words), and even vocal cues (e.g., shaky voice) in the job interview (e.g., DeGroot \& Motowidlo, 1999; Forbes \& Jackson, 1980; Russell, Perkins \& Grinnell, 2008). Specifically, past research (e.g., Hollandsworth, Glazeski \& Dressel, 1978; Levine \& Feldman, 2002) has suggested that interview anxiety may manifest itself in the form of speech disturbances (e.g., stuttering, verbal fillers), socially inappropriate behaviors (e.g., appearing rigid or demonstrating little eye contact), and other nervous jitters (e.g., hands shaking). If anxious interviewees are emitting less effective cues, then it is important to identify those cues that are negatively affecting interviewers' perceptions. This knowledge could benefit interviewees, and career counsellors preparing people for interviews, so that appearing anxious doesn't interfere with accurate communication of one's qualifications during an interview.

The relation between anxiety-related behavioral cues and judgments of anxiety is best conceptualized with the Brunswik (1956) lens model (see Figure 1). The lens model provides a framework to understand how an observer (e.g., interviewer) utilizes information (e.g., vocal cues) when forming judgments about a target (e.g., interviewee). The center of the lens model contains the "cues." Cues to anxiety can be emitted by anxious candidates (the left side of the model), and they can be detected and used by interviewers (the right side of the model). Some anxiety-related cues may be emitted by candidates (e.g., fidgeting) but not

Corresponding author:

Deborah M. Powell

Email: dpowell@uoguelph.ca

Phone: 519-824-4120 ext 52167 
FIGURE 1.

Brunswik's Lens Model

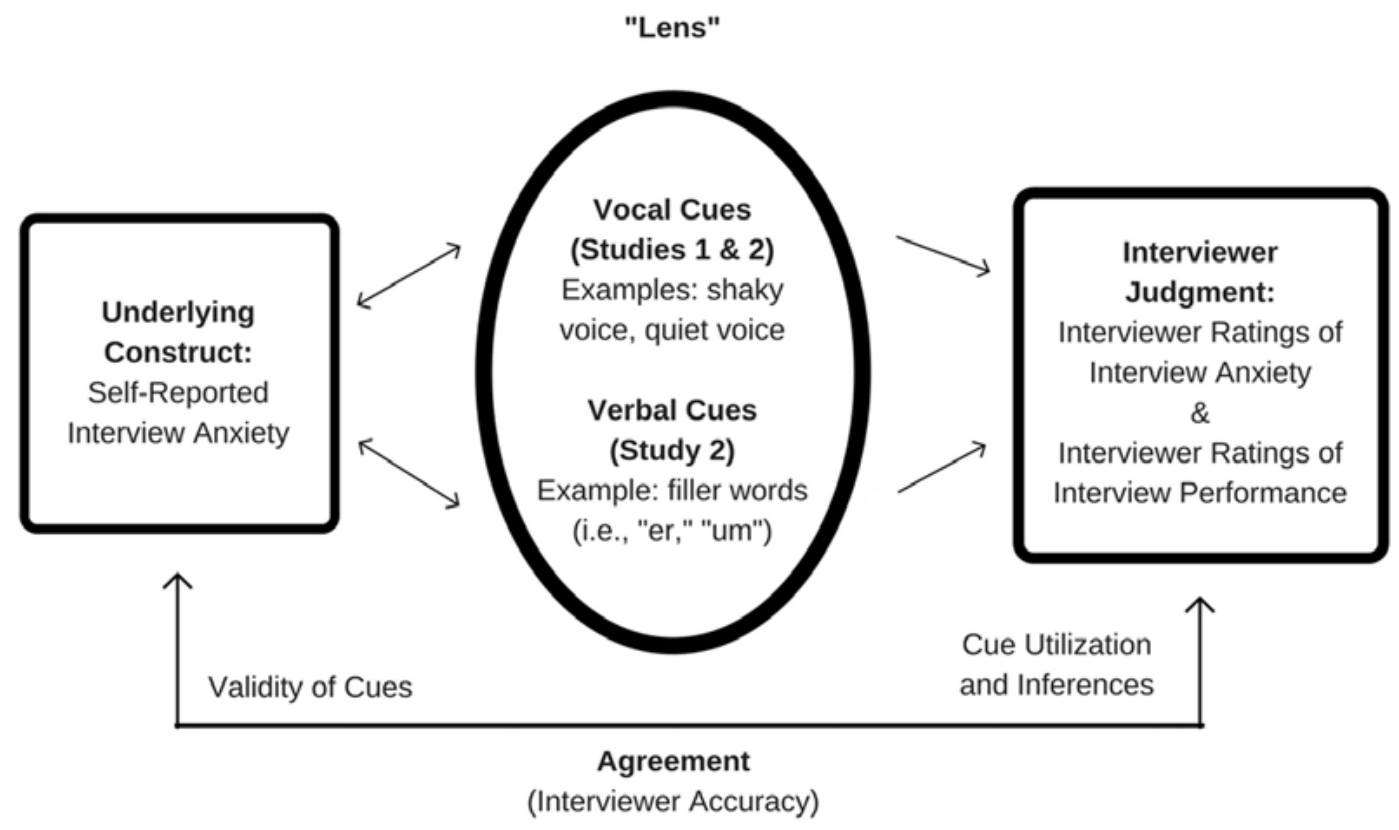

noticed or used by the interviewer. In other cases, cues may actually be unrelated to anxiety (e.g., poor posture), yet they might be used by the interviewer to infer anxiety. The lens model can help to explain how various cues could be accurately, or inaccurately, interpreted when making ratings of anxiety.

There are a variety of different cues to anxiety. For example, vocal or paralinguistic cues refer to the sounds produced by the vocal cords (pitch, volume, and so forth) and often convey supplementary information to a spoken language (Schuller et al., 2013). Many vocal cues are biologically rooted (e.g., the shape of the vocal folds), but some may be purposely enacted, such as a sarcastic tone. In comparison, verbal cues, such as the choice of words, are rooted in a language and have been studied as expressed orally (Argyle, Alkema, \& Gilmour, 1971), as well as in text (Kraut, 1978; Isbister \& Nass, 2000). Finally, nonverbal cues do not involve language but rather include cues such as gaze aversion and body posture (Rasmussen, 1984).

\section{The Present Studies}

The purpose of the present studies was to investigate the role of vocal cues (e.g., shaky voice, quiet voice) in judges' perceptions of interviewee anxiety. We designed an experiment where we restricted the availability of vocal cues by presenting a text-only transcript of an interview; we compared the anxiety ratings made by judges exposed to this text-only transcript to ratings made by judges who heard the audio version. By restricting the available vocal cues, we hypothesized that judges would be less able to de- tect anxiety.

In two studies, we examined the effect of the presence (versus absence) of vocal cues on judges' ratings of interview anxiety and interview performance. In Study 1, we designed an experiment in which participants rated either a high-anxiety candidate or a low-anxiety candidate and were exposed to either an audio version of the interview or a text-only version. In Study 2, we further restricted the available cues by creating a third condition-a text-only version with filler words (um and ah) cleaned out.

We hypothesized that delivery medium (audio or text-only) would interact with level of anxiety (high, low) such that interview anxiety ratings and interview performance scores will differ more strongly within the audio condition. Without the presence of vocal cues, we hypothesized that interview anxiety ratings and interview performance scores will not significantly differ between the high and low anxiety candidates. When more vocal cues are available, participants will be able detect more interview anxiety, and the presence of anxiety-related vocal cues will subsequently lead to lower performance ratings.

Hypothesis 1: Delivery medium (audio or text) will moderate the effect of anxiety condition (high vs. low) on observer ratings of anxiety such that:

$a$. Observer-rated anxiety scores will be higher for the high-anxious candidate within the audio condition;

$b$. Observer rated anxiety scores will not significantly differ across the two candidates within the text-only condition. 
Hypothesis 2: Delivery medium (audio or text) will significantly moderate the relation between level of anxiety (high, low) on ratings of interview performance such that:

a. Observer rated performance scores will be lower for the high-anxious candidate within the audio condition;

b. Observer rated performance scores will not significantly differ across the two candidates within the text-only condition.

\section{METHOD: STUDY 1}

\section{Participants}

We recruited 100 undergraduate students from a Canadian university; 28 participants were removed due to incomplete data or failing an attention-check question ( $\mathrm{n}=$ $72,88.9 \%$ female; average age of $18.68, S D$ 1.27). Details on the attention check items are provided in the Procedure section. Seventy-two percent of the participants were Caucasian; the other $28 \%$ were African American, Asian, Hispanic, or "other." Participants received course credit $(0.5 \%$ on final grade) for their participation.

\section{Interviews (stimulus)}

Two mock employment interviews were selected from a previously collected dataset (Feiler \& Powell, 2015). In that dataset, 125 co-op students from a Canadian university took part in mock employment interviews as a requirement of their co-op course, and these interviews were videotaped. Each interview consisted of approximately six semistructured interview questions that were developed collaboratively by the campus career center and the organizations that were hiring co-op students. Interviews began with general questions (e.g., "tell me a bit about yourself") and then asked more specific questions about the positions the co-op students would be applying for (e.g., "Tell me about a time you couldn't keep on schedule and how did you deal with it?"; "describe one time you've had to adapt to an unfamiliar work environment").

Interviewees who agreed to be in that study filled out a self-report measure of interview anxiety, the Measure of Anxiety in the Selection Interview (MASI; McCarthy \& Goffin, 2004). Sample items from the MASI include: "I became so apprehensive in the job interview that I was unable to express my thoughts clearly" and "In the job interview, I got very nervous about whether my performance was good enough." The original interviewers (who were trained peer helpers) rated interview performance on a 1-100 relative percentile method rating scale (RPM; Goffin, Jelley, Powell, \& Johnston, 2009).

From that dataset of 125 interviews, we rank ordered the participants from least anxious to most anxious (based on self-ratings) so that we could select one interviewee from the low end of anxiety and one from the high end. Then, we selected two interviews for the current study by finding two participants who received the same score on their live interview performance - as rated by their interviewer $(60 / 100)$ yet with different scores on self-rated anxiety (1.47/5 for low anxiety and 3.63/5 for high anxiety). We selected the two interviews based solely on their self-rated anxiety scores and interviewer-rated performance score, without watching the video. Then, we retrieved the video that corresponded to those two participants and made a transcript of each (see Appendix A for the transcripts of the two interviews.) We note that previous research has found a negative correlation between self-reported anxiety and interview performance (e.g., $r=-.19$ in McCarthy \& Goffin, 2004); yet, the two interviews we chose for this study had the same level of performance despite very different levels of anxiety. It may be the case that the more anxious candidate was actually more qualified, or the original interviewers may have used the performance rating scale differently. Despite this limitation, we chose to use actual, unscripted interviews, rather than scripted interviews with actors, so that the cues to anxiety were naturalistic. As well, we chose two interviewees who were initially rated by their live interviewers to have the same level of performance in an attempt to keep performance level constant while investigating cues to the interviewees' self-reported anxiety.

\section{Observer-rated anxiety}

The participants in the current study (students) rated interviewee anxiety levels using a four-item scale that we created for the current study (see Appendix B). The items were rated on a five-point Likert scale ( $1=$ not at all; $5=$ very). This scale had high internal consistency across both studies (Study 1, $\alpha=.78$; Study 2, $\alpha=.87$ ). Participants were also asked to provide a short open-ended response explaining what influenced their ratings of anxiety.

\section{Interview performance}

Participants assessed interview performance using nine items (see Appendix C). Four items were from Stevens and Kristof (1995; e.g., "How qualified is this applicant for the job?"), and five items were taken from a feedback form developed by the on-campus career center (e.g., "The interviewee uses appropriate language"). The questions were rated on a five-point Likert scale $(1=$ strongly disagree; 5 $=$ strongly agree). This scale had high internal consistency across both studies (Study $1 \alpha=.92$; Study $2 \alpha=.90$ ). Participants were also asked to provide a short open-ended response explaining what influenced their ratings of interview performance.

\section{Procedure}

The study was delivered online via Qualtrics. After reading the consent form, the survey allocated the partici- 
pants into one of four experimental conditions (low-anxiety/ text, low-anxiety/audio, high-anxiety/text, high-anxiety/ audio). After participants either listened to or read the interview segment, they completed the interview observer-rated anxiety scale, the performance measure, and the open-ended questions, followed by demographic information.

\section{Attention checks}

We had three different attention checks. First, in order to ensure that participants in the audio condition had functional sound, they were unable to proceed through the survey until they had correctly answered an audio captcha question. The audio captcha randomly recited one of four words (elephant, building, cactus, or headphones) and was answered in multiple choice format (no participants were eliminated at this stage).

Second, participants in the audio condition were unable to proceed through the survey until the full interview segment was played. Alternatively, for participants in the text condition, data were removed if a participant spent less than 90 seconds reading the interview transcripts, resulting in four removed data sets.

Finally, all participants were required to answer one attention-check question regarding the content of the interview. Participants were asked one of two questions depending on which interview they were assigned. Question 1 asked where the interviewee had said they previously worked (a theater, amusement park, factory, or a car dealership), and Question 2 asked where the interviewee gained the majority of their work experience (Walmart, Zellers, Canadian Tire, or Sears). Data were removed if participants answered the question incorrectly, resulting in the removal of 16 data sets.

\section{RESULTS $^{1}$}

The means and standard deviations for each condition are reported in Table 1. In order to test Hypothesis 1, that delivery medium (audio or text) will moderate the effect of anxiety condition on observer-rated anxiety, we conducted a 2x2 factorial ANOVA. The interaction between delivery medium (text, audio) and anxiety condition (high, low) on observer-rated anxiety scores was not significant, $F(1,68)=$ $0.09, \eta^{2}=.001, p=.77$. There was a main effect of anxiety condition, $F(1,68)=5.25, d=0.67[.19,1.15], p=.025$, indicating that participants who rated the highly anxious candidate $(M=3.82, S D=0.84)$ provided higher observer-rated anxiety scores than did those who rated the less anxious candidate $(M=3.30, S D=0.69)$.

Two planned $t$-tests, with false discovery rate corrected $p$-values (FDR; Benjamini \& Hochberg, 1995), were used to investigate the effect of anxiety condition (high, low) on observer-reported anxiety at each level of delivery medium (audio, text). In the audio condition, the high-anxiety candidate was rated as more anxious than was the low-anxiety candidate, $d=0.58[-0.11,1.25]$. However, this difference was not statistically significant, $t(33)=1.70, p=.05$.
The standardized effect size was medium (Cohen, 1988). However, the $95 \%$ confidence interval (CI) is quite long, consistent with anywhere from no difference up to a large difference.

In the text condition, the high anxiety candidate was also rated as more anxious than the low-anxiety text candidate, $d=0.57[-0.15,1.29]$, however this difference was also not statistically significant, $t(35)=1.59, p=.06$. Again, the standardized effect size was medium, but the CI was long, consistent with anywhere from a slightly lower rating up to a higher rating for a the more anxious candidate. Surprisingly, despite the lack of vocal cues in the text-only condition, the difference between the high and low anxiety candidate (in terms of standardized effect size) were similar in both the audio and text conditions.

When examining interview performance as the dependent variable, the interaction between delivery medium (text, audio) and anxiety level (high, low) on ratings of interview performance was not significant, $F(1,68)=0.75, \eta^{2}$ $=.01, p=.39$. There was a main effect of anxiety condition, $F(1,68)=57.49, d=1.93[1.36,2.49], p<.001$, indicating that participants who rated the less anxious candidate $(M$ $=2.91, S D=0.62)$ provided higher interview performance ratings than those who rated the highly anxious candidate $(M$ $=1.81, S D=0.52$ ).

Two planned $t$-tests (with FDR-corrected $p$-values) were used to investigate the effect of anxiety condition (high, low) on interview performance at each level of delivery medium (audio, text). Within the audio condition, the high-anxiety candidate received lower interview performance scores than did the low anxiety candidate, $d=2.44$ $[1.54,3.31], t(33)=-7.19, p<.001$. The standardized effect size is large, and the CI is consistent with participants providing much higher interview performance ratings for the low anxiety candidate. Similarly, within the text condition, the high anxiety candidate received lower interview performance ratings in comparison to the low anxiety candidate, $d=1.48[0.69,2.26], t(35)=-4.13, p<.001$. The standardized effect size was large, however the CI was long, consistent with anywhere from a moderate difference to a large difference. Hypothesis 2, which predicted an interaction between delivery medium (audio, text) and anxiety condition (high, low) on interview performance scores, was not supported. Interview performance scores were found to significantly differ in both the audio and the text conditions.

\section{Qualitative responses}

Participants in Study 1 were asked to provide a brief short-answer response explaining why they provided the ratings they did for interview anxiety. Two coders read through the responses and developed 15 categories of responses. Definitions of each of these categories are described in Table 2. Two different coders then categorized

\footnotetext{
1 The data and $\mathrm{R}$ code for this study can be viewed at https://osf. io/2zpm8/?view_only=36f90eda24fb43dfa8e314e80493b65b
} 
TABLE 1.

Study 1 Descriptive Statistics: Delivery Medium and Anxiety Condition by Dependent Variables

\begin{tabular}{|c|c|c|c|}
\hline Delivery medium & Low anxiety & High anxiety & \\
\hline & Mean $[S D]$ & Mean $[S D]$ & $d[95 \% \mathrm{CI}]$ \\
\hline \multicolumn{4}{|c|}{ Observer-rated anxiety } \\
\hline Audio & $3.21[0.50]$ & $3.59[0.82]$ & $0.58[-0.11,1.25]$ \\
\hline Text & $3.45[0.94]$ & $3.95[0.84]$ & $0.57[-0.15,1.28]$ \\
\hline \multicolumn{4}{|c|}{ Interview performance } \\
\hline Audio & $3.12[0.43]$ & $2.03[0.47]$ & $2.44[1.54,3.31]$ \\
\hline Text & $2.55[0.74]$ & $1.68[0.51]$ & $1.48[0.69,2.26]$ \\
\hline
\end{tabular}

TABLE 2.

Descriptions of Qualitative Coding Categories

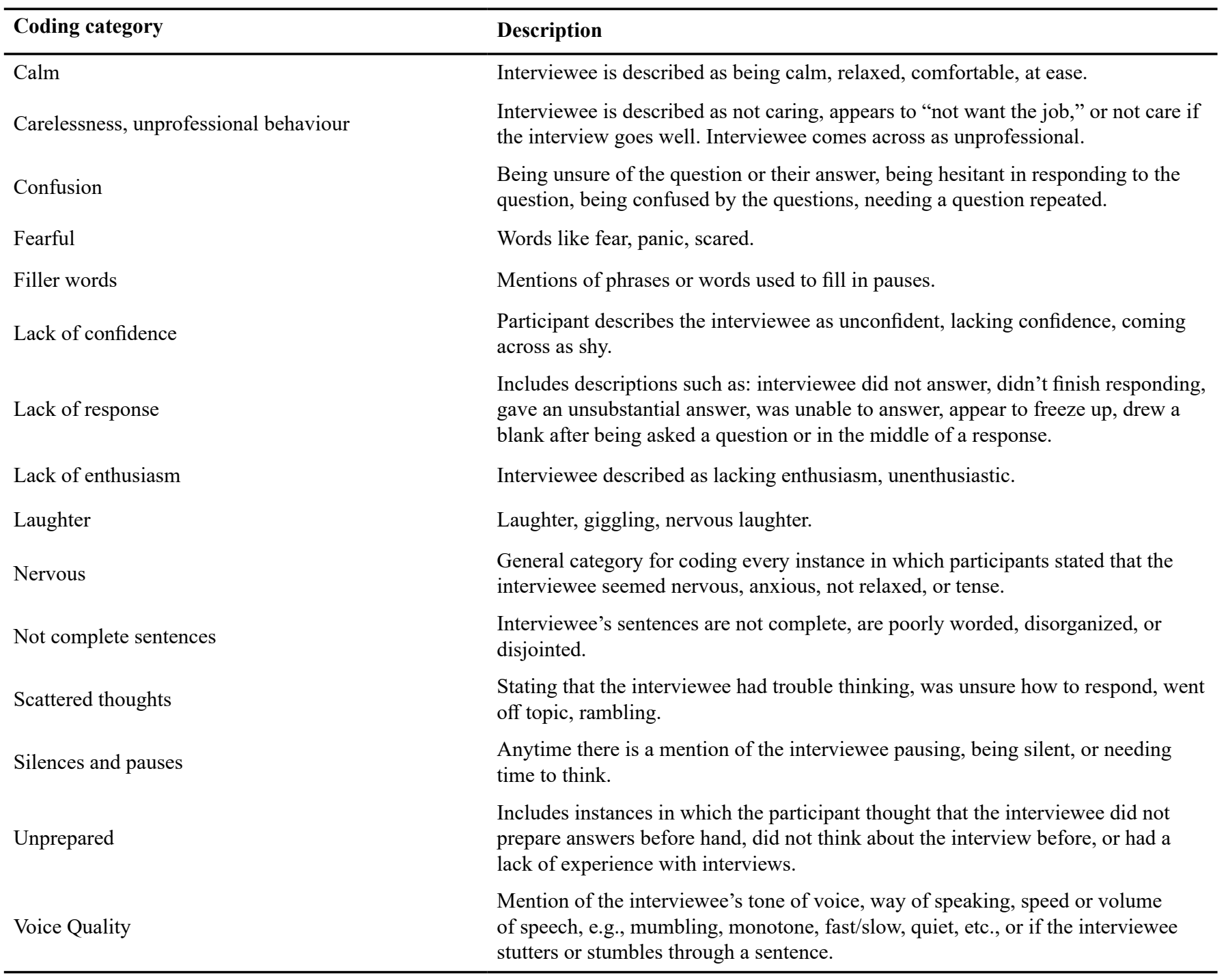


each response according to which categories it fell into (each response could be coded into more than one category.) A summary of the frequencies of each category is shown in Figures 2 and 3. In Figure 2, we display the high anxiety condition (text and audio), and have put the categories in order, from most frequently listed category used in the audio condition, to least frequently used in the audio condition. The corresponding frequency of each cue as used in the text condition is shown in the bar below the audio condition. An examination of these frequencies shows that, for participants in the high anxiety-audio condition, when all vocal cues are available, "voice quality" was the most commonly listed cue to anxiety. Some sample comments about voice quality included:

"She didn't use a confident tone or confident language, she spoke quietly";

"I assume she was nervous/fearful given her very quiet tone";

"Her voice was uneasy."

In contrast, in the high anxiety-text condition, when vocal cues are restricted, "nervous" - a generic response with little detail—was commonly used (e.g., "she did seem really tense, anxious and scared"). When participants listed more specific reasons for their anxiety ratings in the high anxiety-text condition, they most often cited "filler words" as the cue on which they relied. Some sample comments about filler words included:

"I think because they used a lot of filler words and generally spoke in an unprofessional way that they were more nervous";

"Interviewee used an excessive amount of filler words";

"Used "uhm" and other words which comes off as anxious."

These comments about filler words, which were commonly noted in the text condition, seem to suggest that when cues such as "voice quality" are restricted, interviewers may pay more attention to other cues that are available, such as filler words. In addition, filler words such as "um" may become more noticeable when reading a text version of an interview, compared to listening to an audio version.

\section{DISCUSSION}

Across both text and audio conditions, the high-anxiety interviewee was rated higher on observer-rated anxiety and lower on observer ratings of interview performance as compared to the low-anxiety interviewee. Taken together, these findings were surprising, in that they did not provide support for the hypothesis that the restriction of vocal cues (text-only condition) would lead to smaller differences in observer ratings of anxiety and performance as compared to the unrestricted vocal cue (audio) condition.

One potential reason that this study did not show support for the expected hypotheses is that the text-only condition, while restricting vocal cues (pitch, volume), still contained verbal cues to anxiety. In a 2008 study, Russell et al. created simulated interview audiotapes and interjected the fillers of "like" or "uh" throughout the transcript; compared to the no-filler condition, the "like" and "uh" groups were rated as lower on "employability." Indeed, in the qualitative section of our Study 1, several participants noted that the presence of filler words (e.g., um, ah) influenced their rating of interview anxiety. Whereas the use of filler words may come across as part of natural and fluent conversation (when not used excessively), they may stand out in transcripts and therefore influence interview anxiety and interview performance appraisals.

To address this study's limitations, we conducted a second study, which introduced a third level to the delivery medium variable: text-only transcripts with reduced instances of filler words (cleaned text). We also recruited a larger sample for Study 2. Accordingly, Study 2 utilized a 3 (audio, text, cleaned text-only) by 2 (high-anxiety, low-anxiety) design to investigate the influence of filler words in this context.

\section{STUDY 2}

In Study 2, we hypothesized that delivery medium (audio, text, or cleaned text) would interact with level of anxiety (high, low) such that interview anxiety and interview performance ratings will differ more strongly within the audio condition due to the presence of vocal cues in this condition. We expected that the even if participants could detect anxiety in the text condition (as they seemed to in Study 1), they would not be able to do so in the cleaned text condition, because filler words could no longer be used as cues. Without the presence of vocal cues or the verbal cue of filler words for participants to assess, we hypothesized that interview anxiety ratings and interview performance scores would not significantly differ between the high and low anxiety candidates.

Hypothesis 3: Delivery medium (audio, text, or cleaned text) will moderate the effect of anxiety condition (high vs low) on observer ratings of anxiety such that:

a. Observer-rated anxiety scores will be higher for the high-anxious candidate (compared to the low anxious candidate) within the audio condition;

$b$. Observer rated anxiety scores will not significantly differ within the text-only condition;

c. Observer rated anxiety scores will not significantly differ within the cleaned text-only condition. 
FIGURE 2.

Frequency of cues cited in qualitative responses in Study 1: High Anxiety Condition

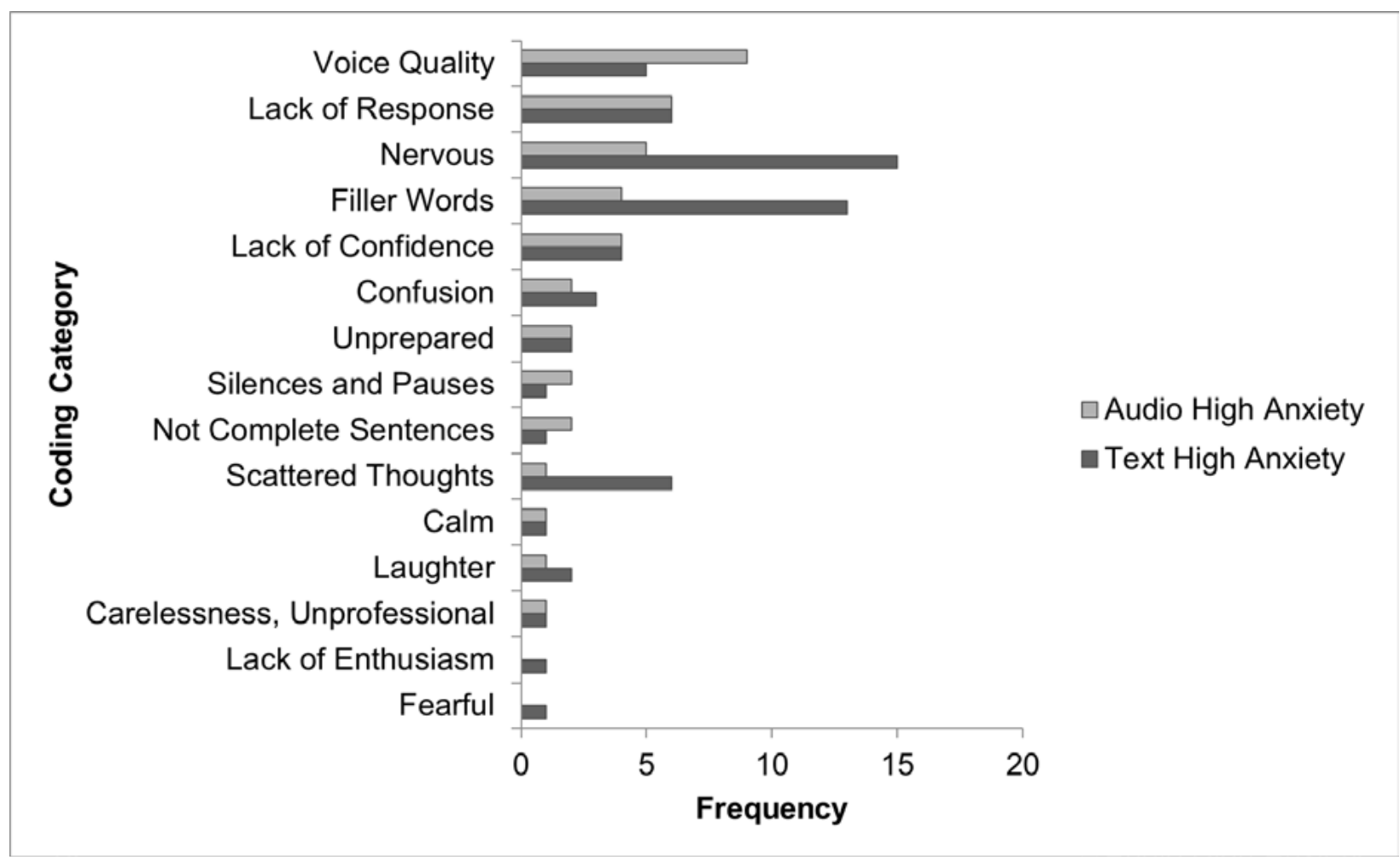

FIGURE 3.

Frequency of cues cited in qualitative responses in Study 1: Low Anxiety Condition

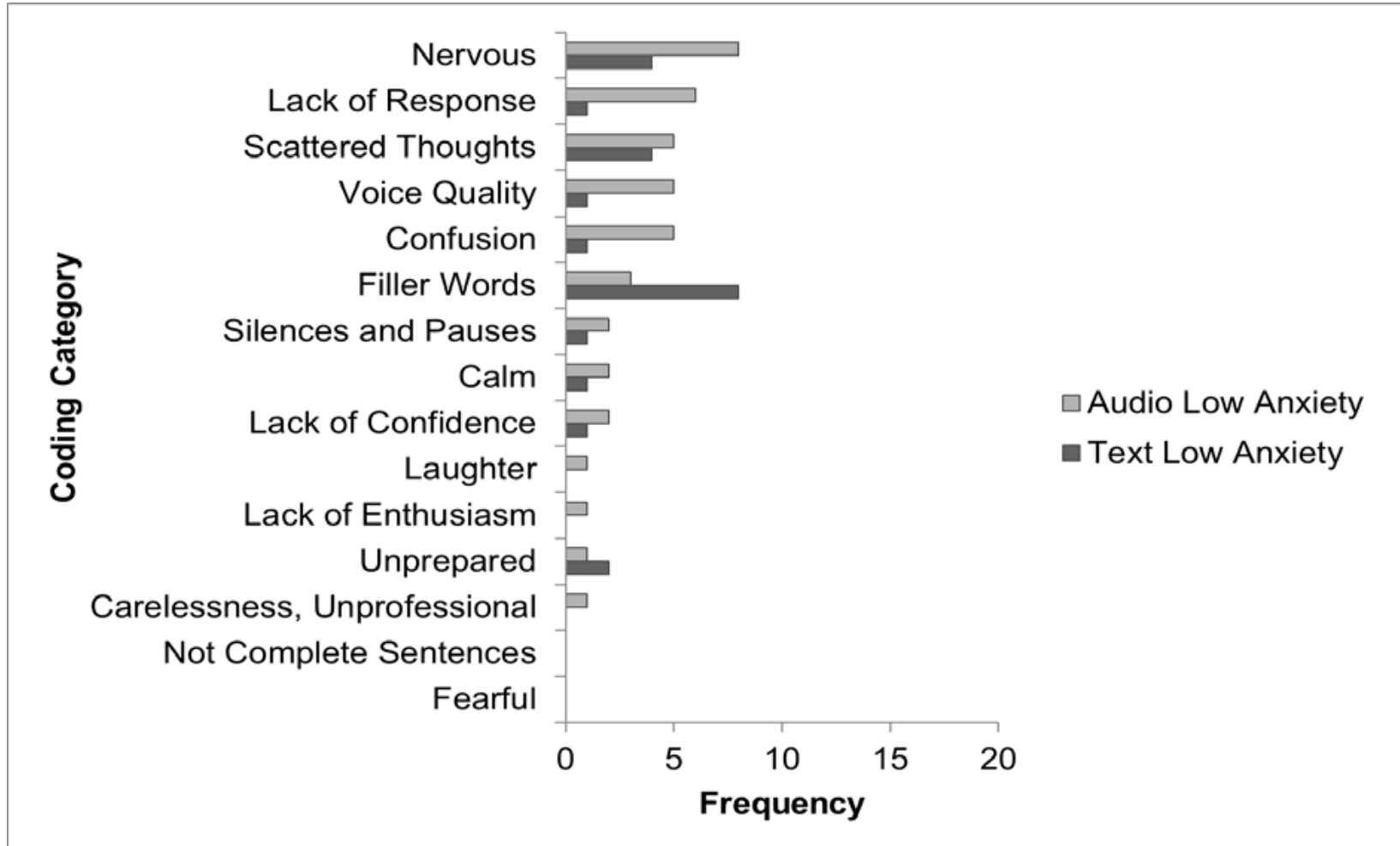


Hypothesis 4: Delivery medium (audio or text) will significantly moderate the relation between level of anxiety (high, low) on ratings of interview performance such that:

a. Observer rated performance scores will be lower for the high-anxious candidate within the audio condition;

b. Observer rated performance scores will not significantly differ within the text-only condition;

c. Observer rated performance scores will not significantly differ within the cleaned text condition.

\section{METHOD}

\section{Participants}

We recruited 653 undergraduate students enrolled in first-year psychology courses at a Canadian university. The data of 242 participants were removed due to (a) incomplete surveys (44 removed), (b) reading the interview transcript in less than 90 seconds (104 removed), (c) answering the attention-check questions incorrectly (55 removed), or (d) spending fewer than 5 minutes (34 removed) or greater than 30 minutes ( 5 removed) on the survey. The final sample resulted in a total of 411 participants $(80.3 \%$ female). The average age of participants was $18.71(S D=2.71)$.

Seventy-eight percent of participants were Caucasian, $10 \%$ were Asian, and $12 \%$ fell into other categories (Hispanic, African-American, Aboriginal). Participants received course credit $(0.5 \%$ on final grade) for their participation.

\section{Measures and procedure}

The materials and procedure were identical to Study 1, with the exception of one additional condition: the cleaned text-condition. For that condition, we removed filler words from both the high-anxiety as well as low-anxiety interview transcripts. Specifically, this involved searching through the transcripts and removing text which was a result of an interviewee saying "um" or "ah." Twenty-four filler words were removed in the high-anxiety transcript, and nine filler words for the low-anxiety transcript. The word "like" was not removed, because it can be used in a substantive context.

\section{RESULTS}

Means and standard deviations for each cell are reported in Table 3. To test Hypothesis 3, that delivery medium and anxiety condition would interact to predict anxiety ratings, we conducted at a $3 \times 2$ factorial ANOVA. There was a significant two-way interaction between delivery medium (audio, text, cleaned text) and anxiety condition (high, low), $F(2,405)=3.39, \eta^{2}=.02, p=.035$.

Three planned $t$-tests (with FDR corrected $p$-values) were used to investigate the effect of anxiety across the three levels of delivery medium (audio, text, cleaned-text). Within the audio condition, the high-anxiety candidate was rated as more anxious than was the low-anxiety candidate, $d=1.13[0.76,1.49], t(131)=6.49, p<.001$. The standardized effect size was large, and the CI is consistent with interview anxiety producing a large effect on anxiety ratings. Within the text condition, the high anxiety candidate was rated more anxious than was the low anxiety candidate, $d=0.48[0.13, .93], t(128)=2.721, p=.004$. The standardized effect size was moderate; however, the CI is long, consistent with interview anxiety producing anywhere from a small to a large effect on anxiety ratings. Within the cleaned-text condition, the high-anxiety candidate was also rated as more anxious than was the low-anxiety candidate, $d=0.60[0.27,0.93], t(145)=3.64, p<.001$. Although the standardized effect is moderate, the CI is fairly long and is consistent with interview anxiety producing anywhere from a small to a large effect on anxiety ratings. The interaction we predicted was that observers would not detect differences in anxiety when reading the cleaned-text condition. What we found was that participants could detect differences between the high and low anxiety candidates in all three conditions, but the difference was larger in the audio condition $(d=1.13)$ than in the other two conditions $(0.48$ and 0.60 respectively). The availability of verbal cues magnified the difference between the two candidates.

To test Hypothesis 4, that anxiety condition (high, low) will interact with delivery medium (audio, text, cleanedtext) to predict interview performance ratings, we conducted a $3 \times 2$ factorial ANOVA. There was a significant twoway interaction between delivery medium and anxiety level on interview performance scores, $F(2,405)=8.57, \eta^{2}=.04$, $p<.001$.

Three planned $t$-tests (with FDR corrected $p$-values) were used to investigate the effect of anxiety across the three levels of delivery medium (audio, text, cleaned-text). Within the audio condition, the high-anxiety candidate received lower interview performance scores in comparison to the low anxiety candidate, $d=1.91[1.50,2.32], t(131)=$ $-11.01, p<.001$. The standardized effect size is large, and the $\mathrm{CI}$ is consistent with participants providing much higher interview performance ratings for the low anxiety candidate. Similarly, within the text condition, the high-anxiety candidate received lower interview performance scores in comparison to the low-anxiety interviewee, $d=1.28[0.90$, 1.66], $t(128)=-7.29, p<.001$. The standardized effect size is large, and the CI is consistent with participants providing much higher interview performance ratings for the low anxiety candidate. Finally, in the cleaned-text condition, the high-anxiety candidate received lower interview performance scores in comparison to the low anxiety candidate, $d=1.80[1.41,2.18], t(145)=-10.91, p<.001$. The standardized effect size is large, and the $\mathrm{CI}$ is consistent with participants providing much higher interview performance 
TABLE 3.

Study 2 Descriptive Statistics: Delivery Medium and Anxiety Condition by Dependent Variables

\begin{tabular}{|c|c|c|c|}
\hline Delivery medium & Low anxiety & High anxiety & \\
\hline & Mean $[S D]$ & Mean $[S D]$ & $d[95 \% \mathrm{CI}]$ \\
\hline \multicolumn{4}{|c|}{ Observer-rated anxiety } \\
\hline Audio & $2.82[0.86]$ & $3.75[0.79]$ & $1.12[0.76,1.49]$ \\
\hline Text & $3.59[0.81]$ & $3.98[0.81]$ & $0.48[0.13,0.93]$ \\
\hline Cleaned text & $3.05[0.90]$ & $3.58[0.89]$ & $0.60[0.27,0.93]$ \\
\hline \multicolumn{4}{|c|}{ Interview performance } \\
\hline Audio & $3.40[0.60]$ & $2.16[0.69]$ & $1.91[1.50,2.32]$ \\
\hline Text & $2.42[0.53]$ & $1.75[0.51]$ & $1.28[0.90,1.66]$ \\
\hline Cleaned text & $2.69[0.59]$ & $1.75[0.45]$ & $1.80[1.41,2.18]$ \\
\hline
\end{tabular}

ratings for the low anxiety candidate. The interaction we predicted was that observers would not detect differences in performance when reading the cleaned-text condition. What we found was that participants could detect differences between the high and low anxiety candidates in all three conditions, but the difference was larger in the audio condition $(d=1.91)$ and the cleaned text condition $(d=1.80)$ compared to the text-only condition $(d=1.28)$.

\section{Qualitative responses}

We used the same 15 categories that we developed for Study 1 and had the same two coders categorize the Study 2 open-ended responses. The frequencies for each category are displayed in Figures 4 (high anxiety) and 5 (low anxiety). As in Study 1, we organized the figure from most frequently cited to least cited cue in the audio condition and include the text and cleaned-text frequencies below.

Similar to Study 1, when looking at the high anxiety-audio condition, the categories of "voice quality" (e.g., "It seemed as though they were fairly nervous as their voice sounded a little shaky") and general "nervous" (e.g., "I felt they were nervous and not very relaxed maybe when speaking to a higher figure") were the most commonly mentioned cues to anxiety.

For the high anxiety-text condition, the general "nervous" category (e.g., "They didn't seem very confident"), as well as "filler words" (e.g., "In my opinion, the word "uhm" means that someone is nervous because they don't know what to say"), were the most commonly cited reasons for giving anxiety ratings.

Interestingly, when the filler words were cleaned out, the participants cited a wider variety of categories of cues that they relied on. Specially, the categories of "nervous" (e.g., "Seemed confident at start but got increasingly wor- ried throughout") "filler words" (e.g., "She used informal words such as "like"), "scattered thoughts" (e.g., "They also had problems recalling key memories which can also be a side affect of anxiety") and "lack of response" (e.g., "After the first couple of questions his/her answers were getting smaller and less detailed, and some questions he/she wasn't even able to answer") were the most frequent in this condition. It appears that when one category of cues is restricted (e.g., voice quality) then participants are still able to detect anxiety - they just rely on different cues. When filler words such as "um" and "ah" are restricted, participants may notice other filler words (e.g., "like"), and they may be more likely to notice that the person's response might be scattered or unorganized.

\section{GENERAL DISCUSSION}

Taken together, the results of Study 1 and Study 2 indicate that participants were able to detect when an interviewee was anxious and rated the more anxious interviewee lower on interview performance, even when vocal cues, and one verbal cue (filler words), were restricted through presenting text and cleaned-text formats. Clearly there is still work to be done to determine exactly which interviewee cues influence observer ratings of anxiety. This continues to be an important avenue to explore; this study found that interviewees perceived as more anxious are also perceived as having performed more poorly in their interviews. However, with the current study design, we cannot determine whether someone who performs poorly in the interview (for whatever reason) is subsequently judged to be anxious or whether appearing anxious leads to the lower performance ratings. We selected these two interviewees because they were matched for performance scores $(60 / 100)$ as rated 
FIGURE 4.

Frequency of cues cited in qualitative responses in Study 2: High Anxiety Condition

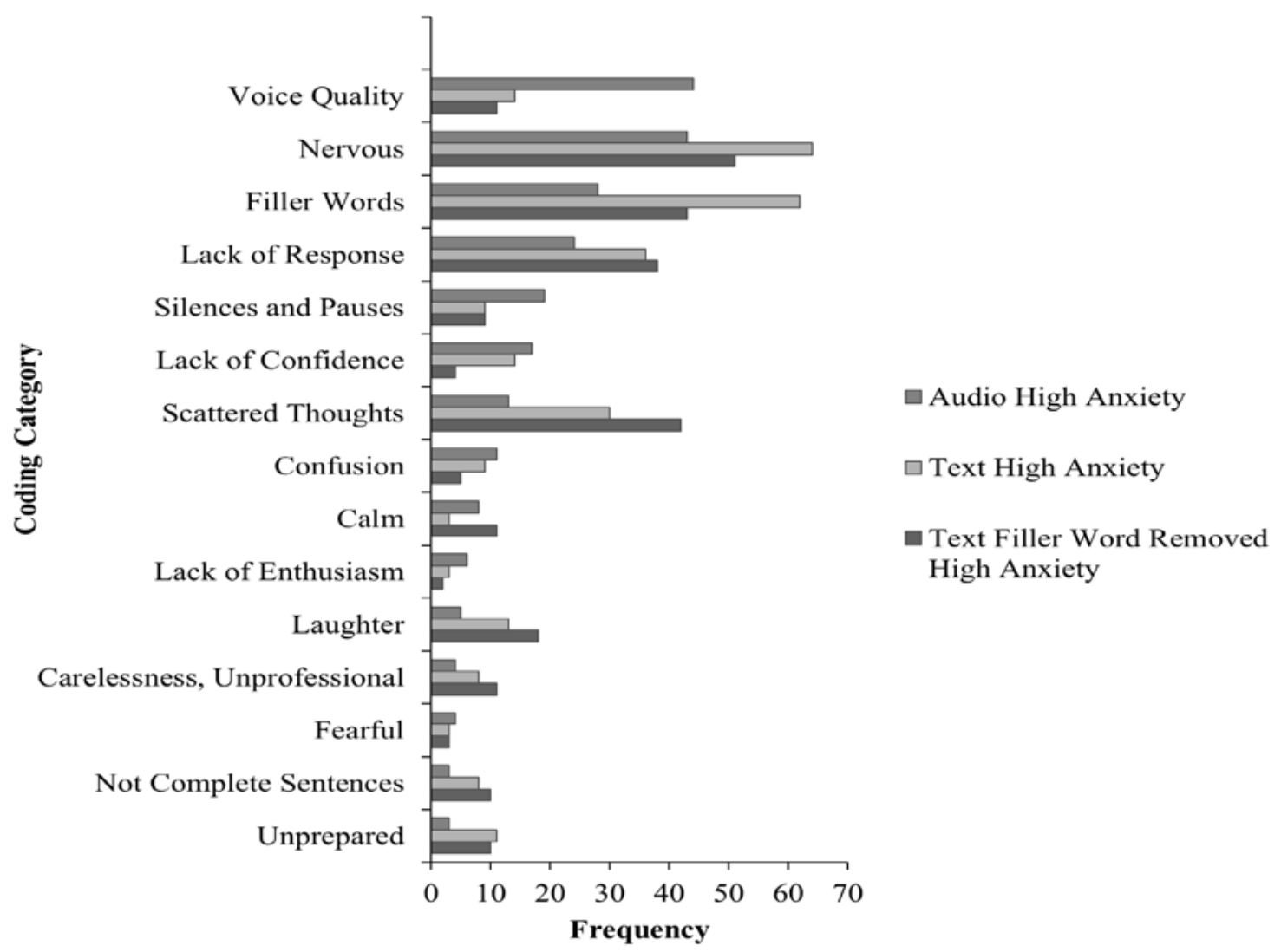

FIGURE 5.

Frequency of cues cited in qualitative responses in Study 2: Low Anxiety Condition

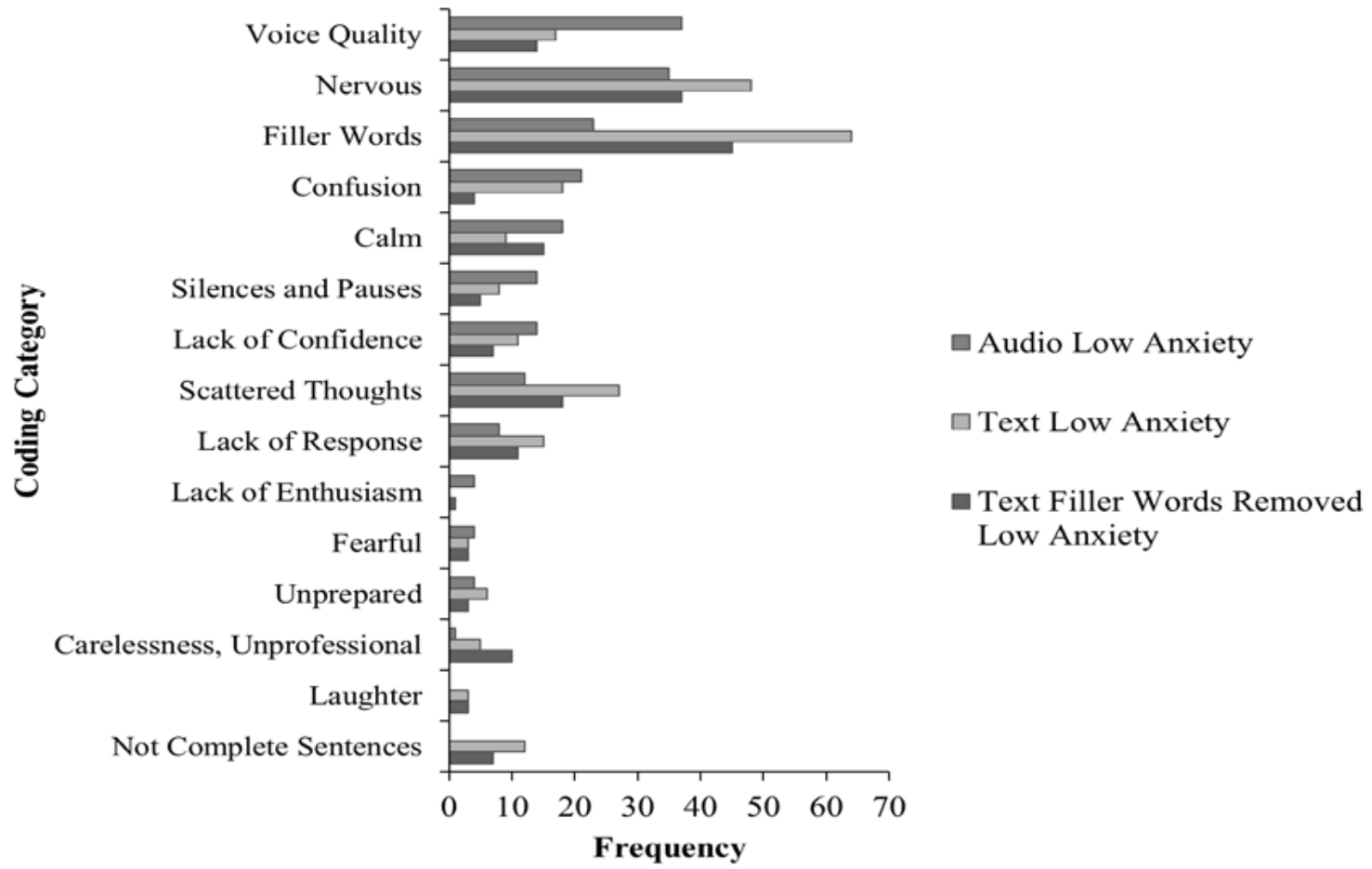


by their original live interviewers: however, they did not receive the same performance scores by the participants in this study. It should be noted that the original live interviewers were peer helpers who were trained on conducting and scoring interviews, whereas the student participants in the current study were not trained interviewers. It appears that in this study, the participants' perceptions of candidate anxiety may have influenced their ratings of candidate performance more than was the case with the original interviewers.

A limitation of the current design is that both of the interviewees were female, and over $80 \%$ of the participants in both studies were female. It may be the case that the results would be different with either male interviewees or a predominately male sample of participants.

The results of the second study are particularly interesting, because participants were able to identify interview anxiety and rated the more anxious interviewee lower on performance despite the reduction of filler words in the cleaned-text condition. Without filler words, and without access to any audio cues (e.g., voice quality), participants were still able to detect interviewee anxiety. The qualitative results of our Study 2 indicated that with the reduction of the most common filler words ("um" and "ah"), participants picked up on other filler words (for instance, participants wrote that the interviewee used the word "like"). It is possible that the absence of some filler words simply redirects attention to other filler words. Further studies should make a concentrated effort to erase all filler words from a transcript to see the effect on ratings of anxiety.

Although we attempted to control filler words, there are a number of other cues that can remain in a transcript. For instance, past studies found that vocal and verbal cues such as words per minute (Feiler \& Powell, 2015), frequency of pauses (Feiler \& Powell, 2015), and power language (Parton, Siltanen, Hosman, \& Langenderfer, 2002) are correlated with ratings of anxiety and performance. In addition, speech errors, such as restarting sentences, repeating words, and unfinished sentences, would still be apparent in transcripts of interviews and in some cases might be even more salient when reading, rather than listening to, an interview. Indeed, in the cleaned-text condition, participants relied on the cue of "scattered thoughts," which could refer to instances where the candidate would switch thoughts in the middle of a sentence one or more times (e.g., "sentences seemed to be stumbled over").

Overall, this study provides evidence that observers can recognize interviewee anxiety, even when specific cues to anxiety are restricted. It appears that when one set of cues (e.g., voice quality) is restricted, then other cues (e.g., filler words or scattered thoughts) become more salient to observers. Future research should continue to explore the ways that vocal and verbal cues to anxiety may manifest themselves in interviews. Career counselling professionals could use this information to coach qualified, but anxious, candidates on strategies to minimize the negative effects of their anxiety on their interview performance. For instance, in the audio version, "voice quality" seemed to be very important cue, including aspects of voice such as mumbling or being monotone. Because it seems to be an important cue, having a confident sounding voice may be an important cue on which to coach interviewees.

\section{REFERENCES}

Argyle, M., Alkema, F., \& Gilmour, R. (1971). The communication of friendly and hostile attitudes by verbal and non-verbal signals. European Journal of Social Psychology, 1, 385-402. 10.1002/ejsp.2420010307

Benjamini, Y., \& Hochberg, Y. (1995). Controlling the false discov ery rate: A practical and powerful approach to multiple testing. Journal of the Royal Statistical Society Series B (Methodological), 57, 289-300.

Brunswik, E. (1956). Historical and thematic relations of psychol ogy to other sciences. Scientific Monthly, 83, 151-161.

Cohen J. (1988). Statistical power analysis for the behavioral sci ences, 2nd ed. Hillsdale, NJ: Erlbaum.

Degroot, T., \& Motowidlo, S. J. (1999). Why visual and vocal in terview cues can affect interviewers' judgments and predict job performance. Journal of Applied Psychology, 84, 986993. doi:10.1037//0021-9010.84.6.986

Feiler, A. R., \& Powell, D. M. (2015). Behavioral expression of job interview anxiety. Journal of Business and Psychology, 31, 155-171. doi:10.1007/s10869-015-9403-z

Forbes, R. J., \& Jackson, P. R. (1980). Non-verbal behaviour and the outcome of selection interviews. Journal of Occupational Psychology, 53, 65-72. doi:10.1111/j.2044-8325.1980. tb00007.x

Goffin, R. D., Jelley, R. B., Powell, D. M., \& Johnston, N. G. (2009). Taking advantage of social comparisons in performance appraisal: The relative percentile method. Human Resource Management, 48, 251-268. doi: 10.1002/hrm.20278

Hollandsworth, J. G., Glazeski, R. C., \& Dressel, M. E. (1978). Use of social-skills training in the treatment of extreme anxiety and deficient verbal skills in the job-interview setting. Journal of Applied Behavior Analysis, 11, 259-269.

Isbister, K., \& Nass, C. (2000). Consistency of personality in inter active characters: Verbal cues, non-verbal cues, and user characteristics. International Journal of Human-Computer Studies, 53, 251-267. doi:10.1006/ijhc.2000.0368.

Kraut, R. E. (1978). Verbal and nonverbal cues in the perception of lying. Journal of Personality and Social Psychology, 36, 380-391.

Levine, S. P., \& Feldman, R. S. (2002). Women and men's nonver bal behavior and self-monitoring in a job interview setting. Applied H.R.M. Research, 7, 1-14. 
McCarthy, J., \& Goffin, R. (2004). Measuring job interview anxi ety: Beyond weak knees and sweaty palms. Personnel Psychology, 57, 607-637. doi:10.1111/j.1744-6570.2004.00002.x

Parton, S. R., Siltanen, S. A., Hosman, L. A., \& Langenderfer, J.

(2002). Employment interview outcomes and speech style effects. Journal of Language and Social Psychology, 21, 144161. doi: 10.1177/02627X02021002003

Rasmussen, K. G. (1984). Nonverbal behavior, verbal behavior, resumé credentials, and selection interview outcomes. Journal of Applied Psychology, 69, 551-556. doi: 10.1037/00219010.69.4.551

Russel, B., Perkins, J., \& Grinnell, H. (2008). Interviewees' overuse of the word "like" and hesitations: Effects in simulated hiring decisions. Psychological Reports, 102, 111-118. doi: 10.2466/pr0.102.1.111-118

Schuller, B., Steidl, S., Batliner, A., Burkhardt, F., Devillers, L., Müller, C., \& Narayanan, S. (2013). Paralinguistics in speech and language-state-of-the-art and the challenge. Computer Speech \& Language, 27, 4-39. doi: 10.1016/ j.csl.2012.02.005

Stevens, C. K., \& Kristof, A. L. (1995). Making the right impres sion: A field study of applicant impression management during job interviews. Journal of Applied Psychology, 80, 587-606. doi:10.1037//0021-9010.80.5.587

RECEIVED 08/04/16 ACCEPTED 10/11/17 
Appendix A

Interview Transcripts

\section{Low Anxiety Transcript}

\section{I: Interviewer \\ P: Participant}

I: Okay, so first off, why did you chose your program at the University of Guelph, why did you choose the University of Guelph in particular, and why the co-op program?

P: I chose to be in Environmental Science 'cause I wanted to have a career that would actually like, make a difference instead of having an English degree, I didn't feel like that would actually do much, and I really like, uhm, learning about the environment and it's really interesting. And I chose the University of Guelph because I've always liked this school, I've come to see my cousin here, it's close enough to home, and it's in an area that I like, it's not too big, and I know that they're like, the animal sciences department are a big thing here, and I chose co-op because I wanted to gain more experience and get more of an idea of what I want to do in graduate school.

\section{I: Alright, what do you know about Barenco?}

P: I know that they're an environmental agency that specialized in working with other companies to improve and rehabilitate things, like, and clean up areas, and with them I know I'd be doing a lot of field work, and like, data collection, and I find that that interesting.

\section{I: Why are you interesting in being an environmental technician with Barenco?}

P: Uhm, I liked this job because it's, I feel like I can learn a lot, and I can also use the skills I have right now, and, in the job description it mentioned there's working with a team, but also as an individual, I really like doing that 'cause I'm very, I'm self-motivated, I like, I like being part of a group, but also I like reading.

\section{I: Okay.}

P: So like I can do that, and it mentioned things about working, like, in the field like outdoors and working inside, so, that would be interesting.

I: Describe your ideal work environment, and then mention specific things that you try to avoid in a job.

P: Ideal work environment would be, I mean like, I'm not sure what you're meaning.

I: So ideal work conditions, or culture, or things like that in the work place so that if you can work in any type of work condition, what kind of place would that be?

P: I'd like to work somewhere that is more hands on than just like, looking at data, I'd like to be able to help collect it too.

\section{I: Okay.}

P: I like a lot of variety.

\section{I: Yup.}

P: And uh, I'd like to be able to learn new things, I don't want a job that would just be like meticulous over and over.

I: Okay, and then what are specific things that you try to avoid in a job?

P: Uhm, I try to avoid taking on too much, so my past jobs I've been in a manager position, and I'm not very good at delegating tasks, sometimes I, I just do it all myself I get , I don't perform as well.

\section{I: Okay.}

P: So I try to avoid that.

I: Yup, alright, describe one time you've had to adapt to an unfamiliar work environment.

P: Uhm, I started doing that at one of my positions at a theater, uhm, actually, at my last job I worked sometimes I worked in the office, and I had to be more professional than I had been in the past, in the past I just work in art studios as an assistant, and in my position in the, I had to work in the office like performing more office tasks so I had to be more professional and watch what I said and dress more professional, and I'd be on-task.

I: Okay, tell me of a situation where you demonstrated initiative and started the ball rolling.

P: Uhm, the job that I worked at last summer at REDACTED, in Mississauga, I was the co-op, or the student coordinator for all the volunteers, and before I came the volunteer program wasn't very established at all, the volunteers were, were like extra students in the class they didn't help much, so, so ever since grade 6 I've worked as an art camp assistant, and so with all that experience I created a whole new guidebook and orientation program for all the volunteers, and I trained and hired and interviewed and scheduled and managed them also, 20 in the past two summers, a new 
thing I had to learn.

I: Okay, okay, and is there anything else about you that I should know?

P: Uhm, I'm very motivated, and I like being challenged and I feel like this position might be for me.

I: 'Kay, do you have any questions for me?

P: Uhm, I was wondering what the, what are the difficulties of past co-op students at Barenco have?

I: Adjusting to field work and acquiring a vehicle for the position, uhm, yeah. Did I answer your question well?

P: Haha.

I: Do you have any other questions?

P: I think that's it.

\section{High Anxiety Transcript}

\section{I: Interviewer \\ P: Participant}

I: Okay, so can you tell me a little bit about your self?

P: Uhm, I grew up in Sarnia my entire life and I just recently moved to Guelph where I go to school at the University of Guelph for Biochemistry, uhm, I've worked at Zellers for 3 years and that's my main work experience, uhm, I am very easy to get along with, I an organized, I've had lots of experience organizing different events which it says on my resume and, uhm, I think I have good teamwork skills which I think that would, uhm, benefit to, if I were to get this position.

I: Mhm. And why did you choose the University of Guelph to go to for your undergraduate degree?

P: Uhm, I chose the University because I have heard many good things about the science programs that go here, and I really like the campus, so.

I: And why did you chose the co-op program over just the regular stream?

P: Uhm, well first of all, I really needed, or wanted to get, uhm, good job experience so that when I'm done, uhm, my studies here that I have like a better chance of getting a job, uhm.

I: And why are you interested in this position with this company?

P: Uhm, I'm interested because one of the main reasons is I'm a, uhm, like to gain professional work experience working in a lab.

I: And can you expand a little bit on your lab skills that you have so far?

P: Uhm, I've, well the main, uhm, skills that I have is working, err, uhm, work, like, in chemistry labs at school.

I: What would you your greatest weakness is, and how has it effected your performance in the past?

P: My greatest weakness would be, uhm, haha, uhm, can I get a second thinking about that?

I: Okay. And this job entails a lot of independent working, can you tell me about a time that you had to complete an assignment with minimal supervision, and what were the major issues and how did you resolve them?

P: I just have to think about it, would we be able to come back to that question?

I: Mhm. Okay so, everyone makes mistakes, can you give me an example of at time at work that you made a mistake, what was the outcome? What steps did you take to rectify the situation? And how would you go about the process differently if it were to happen again?

P: Haha, just have to think.

I: You mentioned you worked at Zellers.

P: Yeah, nothing comes into my head.

I: That's okay, so a successful candidate will need to be flexible, can you tell me about a time you couldn't stay or keep on to schedule and how did you deal with it?

P: Uhm, working at Zellers I had lots of, like, things that I had to get around with like school and stuff, uhm, how I got around with that I, uhm, well I changed my availability to work every single weekend, and then, uhm, that's what I did I guess haha.

I: Uhm, did you have anything else to add, as these are all the questions I have for you?

P: That's it.

I: And did you have any questions?

P: Uhm, no I don't. 
Appendix B

Items on Observer Anxiety Scale

\section{How nervous did this person seem?}

Not at all

Somewhat

Very

1

2

3

4

5

2. How calm and relaxed did this person seem? (reverse coded)

Not at all

Somewhat

Very

1

2

3

4

5

3. How tense did this person appear?

Not at all

Somewhat

Very

1

2

3

4

5

4. How fearful did this person seem?

Not at all

Somewhat

Very

1

2

3

4

5 
Appendix C

Interview Performance Items (rated by study participants)

1. How qualified is this applicant for the job?

Not qualified

Qualified

Very qualified

1

2

3

4

5

2. In your opinion, how attractive would this applicant be to a hiring organization?

Low

Medium

High

1

2

3

4

5

3. How well did this applicant do in the interview?

Poor Moderate Exceptional

$\begin{array}{lllll}1 & 2 & 3 & 4 & 5\end{array}$

4. If you were the hiring organization, how likely would you be to offer him or her the job?

Low Medium High

$\begin{array}{lllll}1 & 2 & 3 & 4 & 5\end{array}$

5. The interviewee avoids the use of filler words (uhm, ah)

Strongly disagree Disagree $\quad$ Undecided $\quad$ Agree $\quad$ Strongly agree

$\begin{array}{lllll}1 & 2 & 3 & 4 & 5\end{array}$

6. The interviewee uses appropriate language

$\begin{array}{lcccc}\text { Strongly disagree } & \text { Disagree } & \text { Undecided } & \text { Agree } & \text { Strongly agree } \\ 1 & 2 & 3 & 4 & 5\end{array}$

7. The interviewee is enthusiastic throughout the interview

$\begin{array}{llll}\text { Strongly disagree } & \text { Disagree } & \text { Undecided } & \text { Agree }\end{array}$

$\begin{array}{lllll}1 & 2 & 3 & 4 & 5\end{array}$

8. The interviewee answered the questions that were asked

$\begin{array}{ccccc}\text { Strongly disagree } & \text { Disagree } & \text { Undecided } & \text { Agree } & \text { Strongly agree } \\ 1 & 2 & 3 & 4 & 5\end{array}$

9. The interviewee's answers to questions are focused.

Strongly disagree Disagree $\quad$ Undecided $\quad$ Agree $\quad$ Strongly agree

$\begin{array}{lllll}1 & 2 & 3 & 4 & 5\end{array}$ 